\title{
The Research and Design of A New Kind of Multi-functional Laser Marking System Based on Embedded Microprocessor
}

\author{
Yafeng $Y u^{1, a}$, Kaijin Qiu ${ }^{1, b, *}$ \\ ${ }^{1}$ College of Computer \& Information Science, Southwest University, Chongqing, China \\ aemail:yyf7329081@126.com, ${ }^{b}$ email:qkjqkj@swu.edu.cn, ${ }^{*}$ corresponding author
}

\begin{abstract}
Keywords: Sensor Technology; Information Collecting and Processing; New Kind of Laser Marking System; Personalization capabilities.
\end{abstract}

\begin{abstract}
The paper designs a new kind of laser marking system which is based on embedded microprocessor with several functions like continuing from the breakpoint, starting from any set point, marking on the curved surface, displaying the trace in real time and so on. The new kind of laser marking system is composed of command module, information collecting and processing module, control module and marking module. The system is improved taking the disadvantages of similar systems off-the-shelf into consideration like poor abilities of dealing with problems on hardware, being unable to communicate and so on. It not only can meet different requirements of various users but is also more user-friendly, more stable and safer. So it is of great practical value.
\end{abstract}

\section{Introduction}

In recent years, with the improvement of technology of using the laser and the rapid development of computer technology and improvement of the optical device, laser marking technology has developed rapidly. However, laser marking machines on the market has the following defects: First, they are not able to start to print from anywhere users want. Second, they can't display the trace in real time. Third, they can't continue form the breakpoint. Last, they don't have the ability of fault detection. And these problems have negative effect on the practicability, reliability and security and bring great troubles and inconvenience to the user with no doubt. Taking these shortcomings into consideration, we design a new laser marking system in this paper which can continue from the breakpoint, start from anywhere, print on a curved surface, detect faults and display the trace. It not only can meet different requirements of various users but is also more user-friendly, more stable and safer.

The new kind of laser marking system in this paper rely mostly on the communication between the communication-micro-controller-unit (communication-MCU) and control-micro-controller-unit (control-MCU) and collecting and processing of information from various sensors.

Literature [1] studies the algorithm and the concrete process for how to express a point in space by coordinate(x,y,z), which provides a reference for processing the data from sensors used to measure the distance from the tip to the surface(distance-measurement-sensor) and position sensor. The framework of the system is based on the example provided by Literature [2], [3], [4] and [5]. The examples illustrated in Literature [2], [3] and [4] all uses a personal computer, a MCU and storage form the marking system to control the marking machine to mark in different ways while the example in Literature [5] adds position sensor and negative feedback circuit to the system to make it more accurate and reliable. At the same time, we also use the communication-MCU to complete most work PC did to improved the original framework, simplifying the work users should do on PC.

The working principle of this new kind of laser marking system is: First, the command is sent from upper computer to communication-MCU combined with information from sensors and then control-MCU produces the control signal after calculating in the communication-MCU. After that, the control signal is sent to the control-MCU through FIFO Memory to control the marking machine. Second, the control-MCU collects the data from the position sensor so that it can send the coordinate of the tip to the communication-MCU, and then to the upper Computer, detecting the 
trace in real time. Third, the control-MCU collects and processes the data from the fault detection sensor to order the Flash storage to power down and save the coordinate of the breakpoint automatically when a fault occurs so that the marking machine can continue from the breakpoint after the fault is cleared. From the principles above, this new kind of laser marking system in this paper, compared with common systems, has new functions of continuing from the breakpoint, starting from any set point, marking on the curved surface, displaying the trace in real time and so on.

The paper designs a new kind of laser marking system, which overcomes the disadvantages of traditional marking systems, and has the following features:

- As for the functions: Because of the new functions of continuing from the breakpoint, starting from any set point and marking on the curved surface, users can choose the working environment(curved surface or flat surface) and set the coordinate of starting point, making the system more user-friendly and flexible.

- As for the structures: Users can command on the PC and the interaction interface is easy enough to use. By the way, transmission rate of USB2.0 up to $480 \mathrm{M} / \mathrm{s}$ so that data can be sent and received in time.

- Displaying the motion trail: Users can check the process of marking by the track displayed on the PC so that users can find the mistake during the process; Fault detection: It can stop automatically when a fault is found and continue from the breakpoint after the fault is cleared.

- Laser marking uses laser to burn the surface, leaving a mark to carve. It is widely used as it can be used for various materials. Also, higher precision, safer working process and longer service life make it more popular.

\section{Structural Design of the new kind of laser marking system}

As is illustrated in Fig.1, the marking system is composed of command module, information collecting and processing module including USB interface, communication MCU, FIFO buffer, distance measurement sensor, position sensor and fault detection sensor, control module which contains control MCU and Flash memory and marking module.

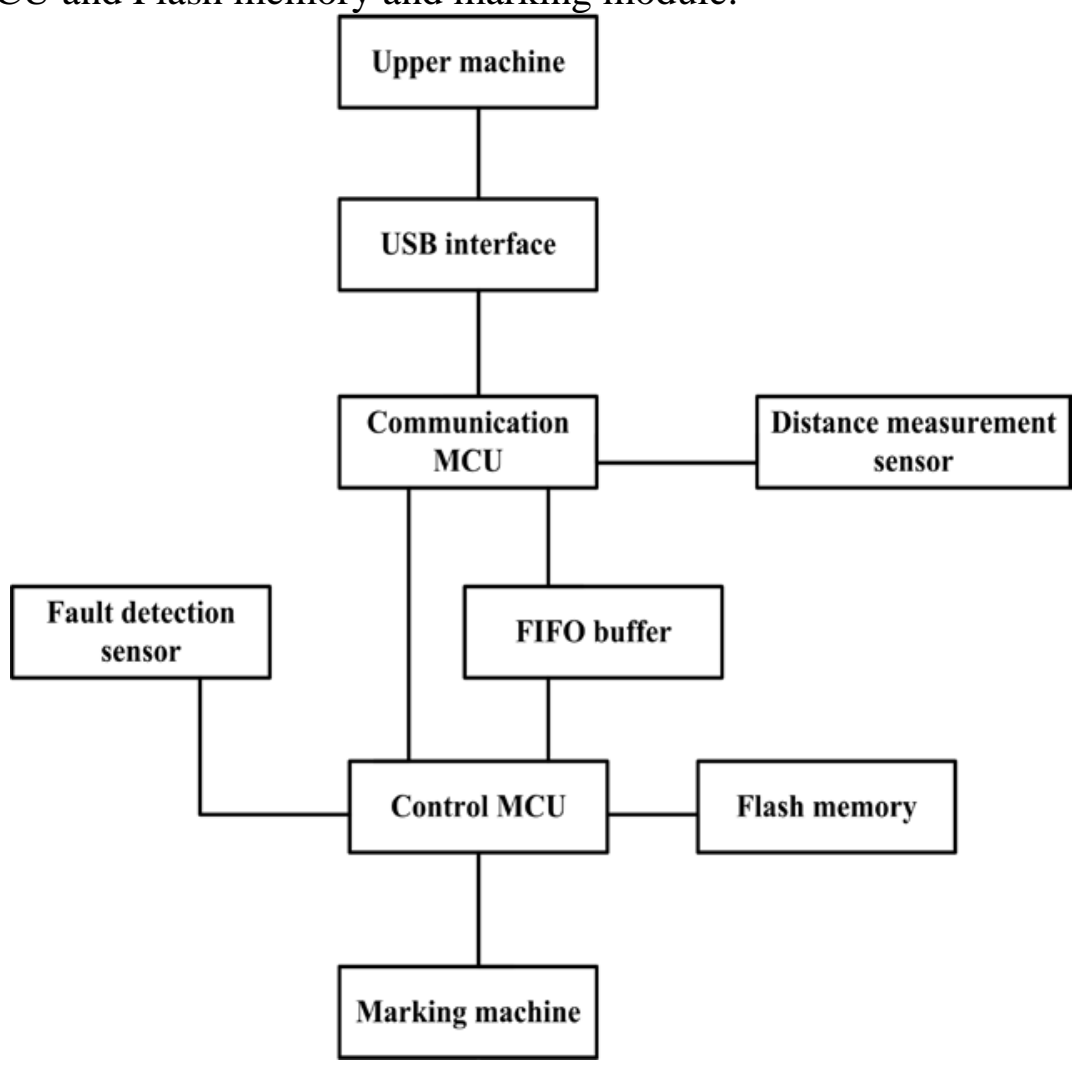

Fig.1. System Structure 
Upper machine: It is used for users to command on the PC so that they can choose the start point and the operating mode, and control the system to start or stop. The PC sends orders to the control MCU through USB interface and communication MCU to control the machine to mark. At the same time, all the orders are numbered so that the track and the position can be displayed on the PC once an order is sent.

USB interface: It adopts Cypress EZ-USB whose transmission rate up to 480M/s so that data can be sent and received in time.

Communication MCU: It is used for the transmission of data and orders and collecting and processing of information from sensors. First, it sends orders from the PC to the control MCU to control the machine to mark. Second, data and information of marking machine are sent from control MCU to communication MCU, and then be sent to upper machine. When a fault is found by sensor, the control MCU will try to calculate to get a control signal based on the track of the tip and data from the sensor to clear the fault. Otherwise, the system will stop until the fault is cleared and save the coordinate of breakpoint to Flash memory which can power down to protect itself, and the coordinate will be sent to the control MCU and marking machine continues to mark from the break point after the system is powered up again. Last, when marking on the curved surface, distance-measurement-sensor will compare the distance between the tip and the surface with standard distance which is the best distance for marking to control the tip to rise or fall to mark.

The communication MCU mentioned above adopts Cortex-M0 microprocessor. Compared with 8051 microprocessor, it is an efficient, low-power and high cost-effective 32-bit MCU. At the same time, taking the case into consideration that this system don't need so many I/O ports, Cortex-M0 microprocessor is the best option for communication MCU.

Distance-measurement-sensor: It is used for collecting the information about the distance between the tip and the surface when marking on the curved surface. It adopts UMT30-1300 A-IUD-L5 Betty ultrasonic sensors which can work at $-25 \sim 70^{\circ} \mathrm{C}$ and under $12 \sim 30 \mathrm{~V}$ DC. It can measure the distance from $200 \mathrm{~mm}$ to $1300 \mathrm{~mm}$ accurately and its resolution reaches $1 \mathrm{~mm}$.

FIFO memory: It is used to connect communication MCU and control MCU and works as a way so that orders can be sent from communication MCU to control MCU. Because of this, data from control MCU to communication MCU can be sent through another way, making the speed of transmission between these two MCUs faster. It adopts IDT7132 dual port RAM chips which is good enough to meet the requirements of the system and cheap at the same time.

Control MCU: It is used to control the tip to move and the laser pen to work. It gets the orders from communication MCU and controls the marking machine to work and sends the position of the tip to communication MCU and the PC. Additionally, It collects the data from fault detection sensor and other information to get the control signal to clear fault and save the coordinate of breakpoint to flash memory to continue from the breakpoint after that.

It adopts STC11f32 microprocessor whose speed is 8 12 times higher than 8051 microprocessor. It has two 16-bit timer, one standalone baud rate generator and one standalone general full-duplex asynchronous serial port. It not only can meet the requirements, but is also cheap.

Fault detection sensor: It judges whether a fault occurred by collecting the data about oscillations when the system is working. It adopts MPU-6000(6050)angular-rate sensor which is small, light and cheap and can detect accurately high-frequency oscillations.

Marking machine: Laser marking uses laser to burn the surface, leaving a mark to carve. It is widely used as it can be used for various materials and higher precision, safer working process and longer service life also make it popular.

\section{System Software Design}

This flow of the system is formed mostly by two parts, communication MCU's collecting and processing of information and control of control MCU. The whole flow of the software is illustrated in Fig.4. 


\section{A. Set Before Working}

First, users should power up the new kind of laser marking system and connect it to the PC. After that, upper machine should be opened and operating mode should be chosen on the human-computer interaction interface which is based on whether the surface you want to mark on is flat. Then, you are supposed to input the coordinate of the point you want to start to mark from. Finally, the figure to be marked on the surface should be upload and users should click the button which represents "start". The system begins to work and the track of marking is displayed in real time on the upper machine. The flow of the software is illustrated in Fig.2.

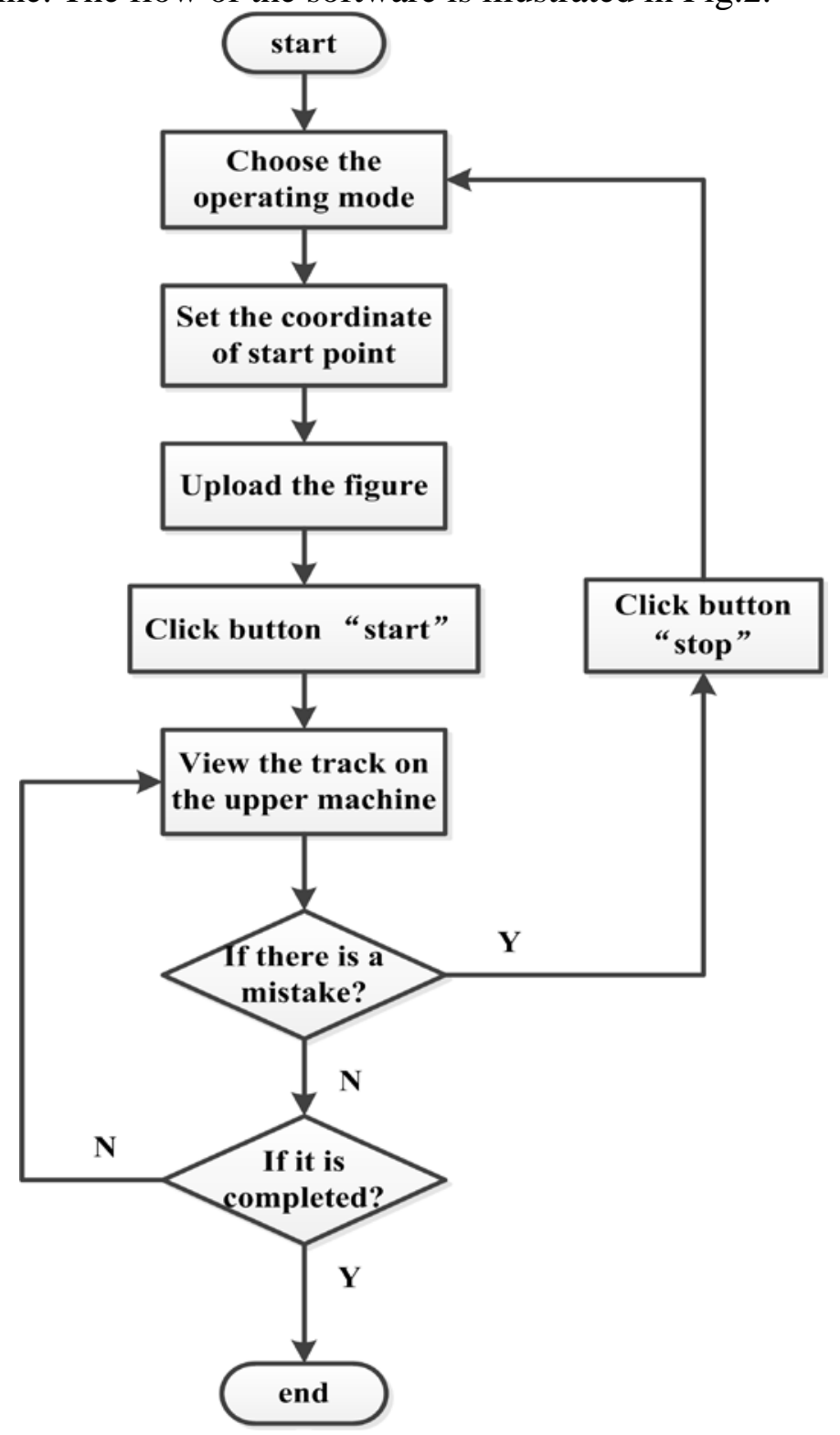

Fig.2. Flow of set before working

\section{B.Operations When A Fault Occurs}

When a fault occurs, system can judge whether it is able to give the order to clear the fault, if not, it powers down automatically and saves the position of the breakpoint, the upper machine should display that the track stops meanwhile. The system continues to mark from the breakpoint and the track on the upper machine update again after the fault is cleared, user powers up the system and the button representing "start" is clicked. The flow of the software is illustrated in Fig.3. 


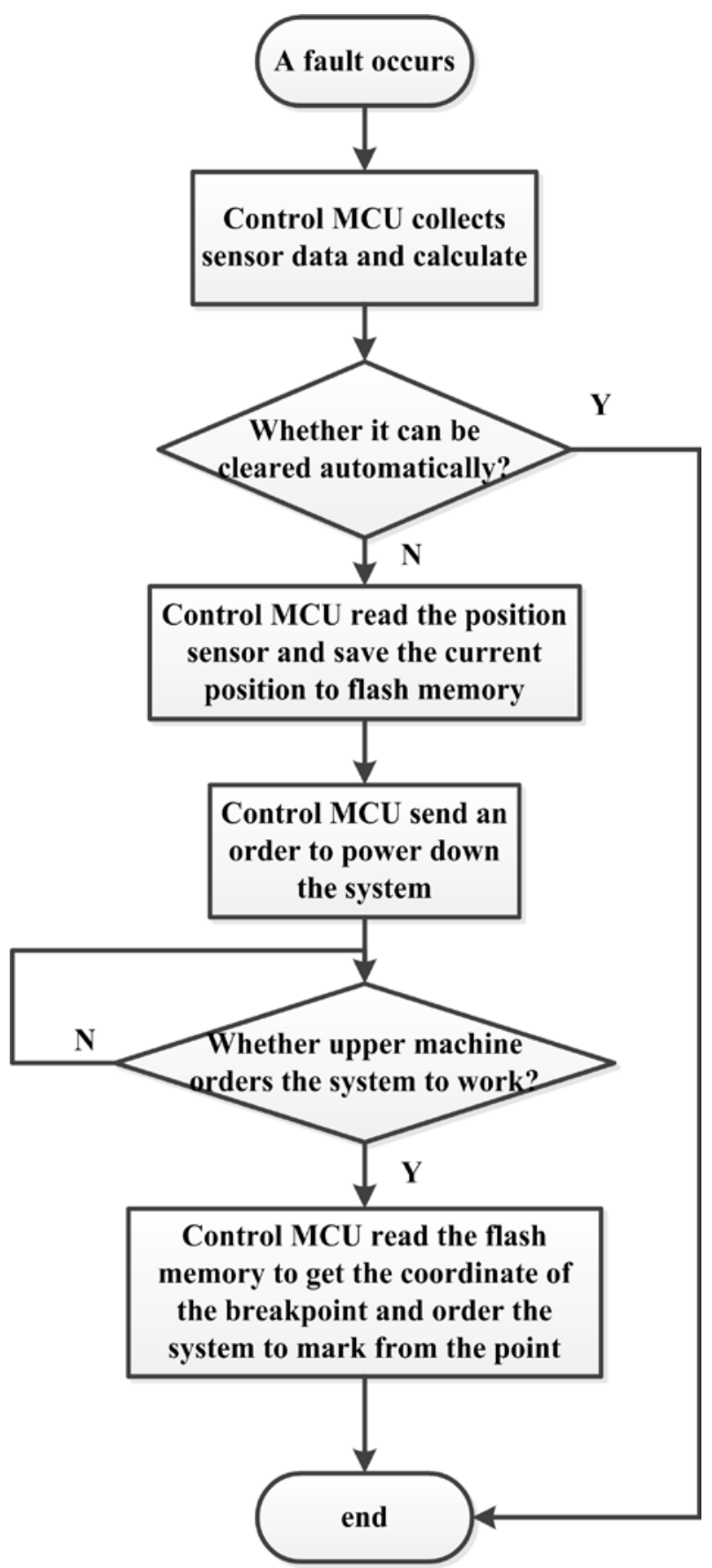

Fig.3. Flow of operation when a fault occurs

\section{C.Operations During Marking}

The system stops marking when button representing "pause" is clicked and position of the breakpoint is saved at the same time, and it continues from the breakpoint to mark when the button representing "start" is clicked during the process of marking. Additionally, there is no doubt that it can be finished by clicking the button representing "stop" so that we can set parameters and begin the next task. 


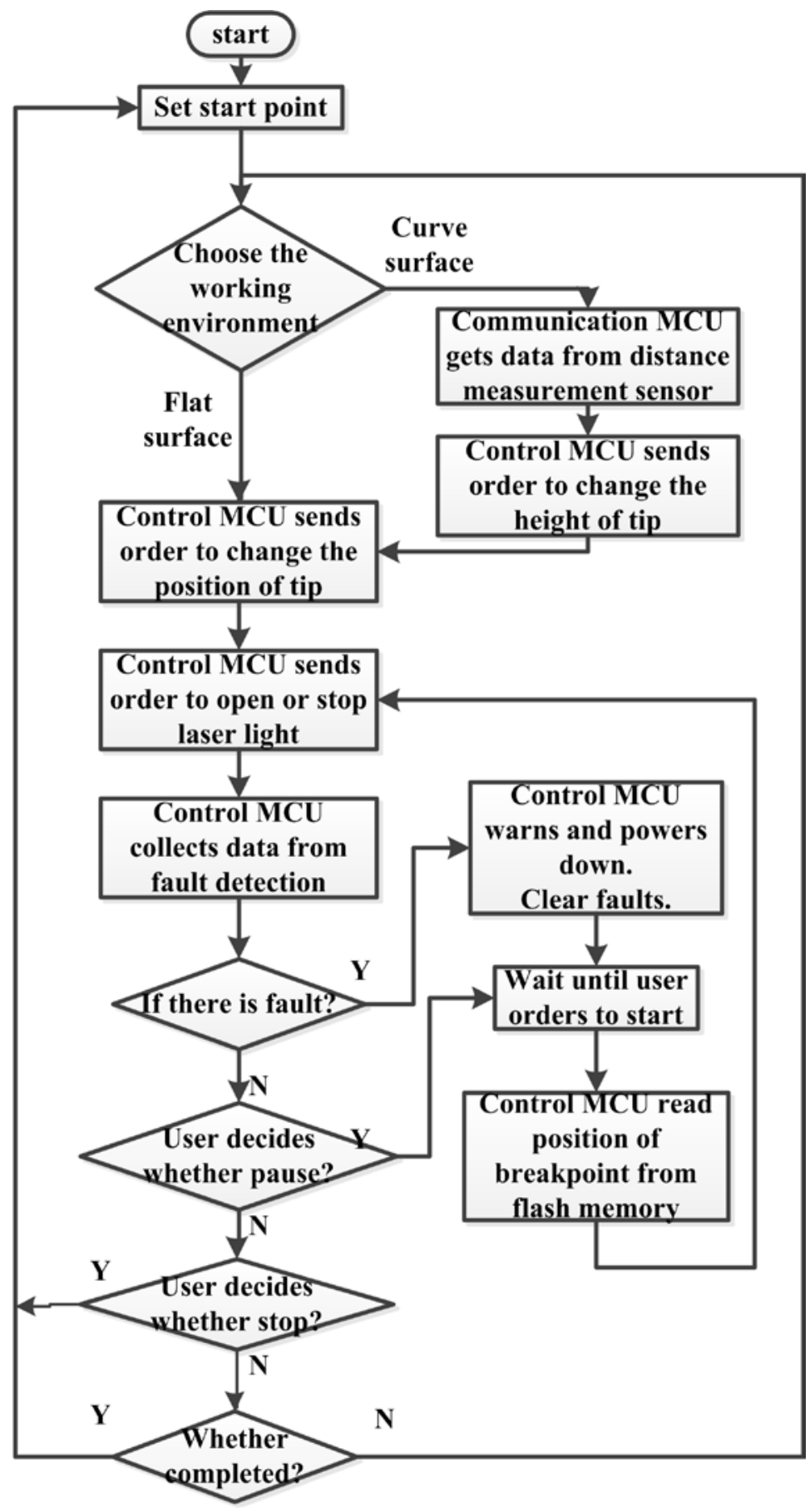

Fig.4. Flow of the laser marking system

\section{Conclusion}

The new kind of laser marking system in this paper, compared with common systems, has new functions of continuing from the breakpoint, starting from any point, marking on the curved surface, displaying the trace in real time and so on. It not only can meet different requirements of various users but is also more user-friendly, more stable and safer. In addition, it should be emphasized that the system is a small-size home marking system with low cost and high practicability and it has a vast market prospect because of this.

Based on the analysis of the mechanical theory as the foundation, designed the soccer robot pick the ball institutions optimal design process, found aim function, select design variables and the corresponding optimization algorithm to optimize a complete set of institutions. At last through the test to get the final performance parameters of the institution. Experiments show that the system has higher accuracy and stability, the new optimize pick the ball have design basic requirements, and 
achieved good ideal control effect.

\section{Acknowledgement}

This work is partially supported by the Augmented Reality Scene of Blankness-Actuality Content Knowledge Representation and Understanding Fusion Technology (cstc2012ggC40004).

\section{References}

[1] Erbao Du, Liandong Yu, A Study on Structure Design and Athematical Model of New Coordinate Measuring Machine [J], Advanced Display. 124th issue, 2011.

[2] Yi Chang, Ning Tan, The Research and Design of Controller of The Laser Marking System Based on Single Chip Microcomputer [J], microcomputer information, 24th volume,5-1 issue, 2007.

[3] Yi Xiao, Shaowen Zhu, Dabin Zhang, Xujun Chen, The Research and Design of a Laser Marking System Controlled by a Computer [J], Computer Measurement \& Control, 5th issue, 2001.

[4] Qiang Hu, Application of M16C62 SCM in Laser Marking System [J], Mechanical manufacture and Automation Major, 6th issue, 2006.

[5] Jianxin Wu, A Design of A New Kind of Laser Marking System [J], Electronic Component \& Device Applications, 10th issue, 2007. 